\title{
Projekt abgebrochen, Folgeband nicht erschienen. Unvollendete Werke in der Wissenschaftsgeschichte der Geschichtswissenschaft
}

\author{
Wolfgang E. J. Weber
}

Angenommen: 27. April 2021 / Online publiziert: 8. Oktober 2021

(C) Der/die Autor(en) 2021

Zusammenfassung Die Konkurrenz zwischen Wissenschaftstheorie (Historik) und Wissenschaftsgeschichte der Geschichtswissenschaft scheint heute zugunsten der Wissenschaftsgeschichte entschieden. Dort herrscht allerdings zumindest in Deutschland noch der individualbiografische Ansatz vor. Wie innovativ dennoch auch dieser Zugriff sein kann, belegt die einschlägige jüngste Monografie von Heinz Duchhardt. Sie befasst sich mit den inneren und äußeren Ursachen nicht zustande gekommener oder abgebrochener Geschichtswerke und dem psychisch-ethischen Umgang der verantwortlichen Historiker mit ihrem „Versagen“. Das Review Essay stellt diese Perspektive kritisch in den übergreifenden wissenschaftshistorischen Kontext und macht weiterführende Forschungsvorschläge.

Schlüsselwörter Geschichte der Geschichtsschreibung - Geschichtswissenschaft . Unvollendete Projekte und Werke · Fachethik - Geschichtswissenschaftliches Rezensionswesen

\section{Project Aborted, Follow-up Volume Not Published. Unfinished Works in the History of Historiography and Historical Science}

\begin{abstract}
The debate on how to best approach the history of history between the theory of historiography (Historik) and the history of the science of historiography (Geschichte der Geschichtswissenschaft) seems to be decided in favour of the history of historiography. Heinz Duchhardt's recent book demonstrates the strength of an individual biographical approach to this field. It deals with the causes of failed or aborted historical works and discusses how the historians dealt with their 'failure' psychologically and ethically. The essay looks at the place of Duchardt's study in
\end{abstract}

Wolfgang E. J. Weber $(\bowtie)$

Institut für Europäische Kulturgeschichte, Universität Augsburg, Augsburg, Deutschland

E-Mail: wolfgang.weber@iek.uni-augsburg.de 
the overarching context of the history of the science of historiography and offers further research suggestions.

Keywords History of Historiography - Historical discipline - Aborted projects and works $\cdot$ Scholarly ethos $\cdot$ Historical reviews

Seit ungefähr einem Jahrzehnt scheint entschieden, dass nicht die Wissenschaftstheorie (Historik), sondern die Wissenschaftsgeschichte den ergiebigsten Zugang zur kritischen Selbstreflexion und Optimierung der Geschichtswissenschaft bietet. Auf der Grundlage dieser Erkenntnis erfährt dieses begrifflich etwas sperrig zu fassende Spezialgebiet historischer Forschung deutlich gesteigerte Beachtung. Allerdings folgen die meisten einschlägigen Beiträge zumindest im deutschen Wissenschaftsbereich bisher eher konventionellen Erkenntnisinteressen, Fragestellungen und Analysemustern. Vor allem ist bemerkenswert, dass nach wie vor die individualbiografische Perspektive dominiert. Das gilt für die Editionen - Korrespondenzen einzelner Historiker - ebenso wie für die Darstellungen, wovon eine Reihe jüngerer Monografien und Sammelbände Zeugnis ablegt. ${ }^{1}$

Dass dennoch auch in diesem, mit dem Vorzug detailfreudig vertiefter Analyse wie dem Nachteil nur punktueller Komparatistik verbundenen Rahmen gewichtige Innovationen möglich sind, stellt die jüngste einschlägige Untersuchung des emeritierten Mainzer Frühneuzeithistorikers Heinz Duchhardt unter Beweis. ${ }^{2}$ Ihr geht es um das Phänomen nur angekündigter, lediglich ideell, bereits konzeptionell oder schon praktisch in Angriff genommener, aber dann nicht abschließend realisierter zweiter (oder sogar dritter) Bände ambitionierter Werke, die von der interessierten Öffentlichkeit, der historisch-kulturwissenschaftlichen ,Zunft‘ und den jeweiligen Autoren zumindest in einer bestimmten Lebens- oder Arbeitsphase als wesentlich

\footnotetext{
1 Exemplarisch Kraus, Hans-Christoph (Hrsg.): Fritz Harting. Korrespondenz eines Historikers zwischen Kaiserreich und Nachkriegszeit, Duncker \& Humblot, Berlin 2019; Dunkhase, Jan Eike (Hrsg.): Reinhart Koselleck - Carl Schmitt. Der Briefwechsel 1953-1983, Suhrkamp, Berlin 2019; Zielinski, Herbert (Hrsg.): Johannes Haller und Karl Straube. Eine Freundschaft im Spiegel der Briefe (Studia Giessensia, NF., Bd. 5), Olms, Wiesbaden u. a. 2018; Duchhardt, Heinz (Hrsg.): Mainzer Historiker (Beiträge zur Geschichte der Universität Mainz. Neue Folge Bd. 16), V\&R unipress, Göttingen 2020; Hruza, Karel (Hrsg.): Österreichische Historiker. Lebensläufe und Karrieren 1900-1945, Bd. 1-3, Böhlau, Wien u. a. 2008-2019; Duchhardt, Heinz: Eine Karriere im Zeichen der Umbrüche. Der Historiker Martin Göhring (1903-1968) in seiner Zeit, Franz Steiner, Stuttgart 2018; Granda, Jeanette: Hermann Kellenbenz (1913-1990). Ein internationaler (Wirtschafts-)Historiker im 20. Jahrhundert, Berliner Wissenschaftsverlag, Berlin 2017; Heinzel, Reto: Theodor Mayer. Ein Mittelalterhistoriker im Banne des „Volkstums“ 1920-1960, Schöningh, Paderborn 2016; Kedar, Benjamin Z./Herde, Peter: Karl Bosl im „,Dritten Reich“, De Gruyter Oldenbourg, Berlin 2016; Berg, Matthias: Karl Alexander von Müller. Historiker für den Nationalsozialismus (Schriftenreihe der Historischen Kommission bei der Bayerischen Akademie der Wissenschaften Bd. 88), Vandenhoeck \& Ruprecht, Göttingen 2014; Nonn, Christoph: Theodor Schieder. Ein bürgerlicher Historiker im 20. Jahrhundert, Droste, Düsseldorf 2013; Dunkhase, Jan Eike: Werner Conze. Ein deutscher Historiker im 20. Jahrhundert (Kritische Studien zur Geschichtswissenschaft, Band 194), Vandenhoeck \& Ruprecht, Göttingen 2010; vgl. zu den Historikern im Nationalsozialismus Link, Fabian: Neuere Entwicklungen in der Wissenschaftsgeschichte: Die (mindestens drei) Wissenschaftskulturen und ihre Historiografien, in: Neue Politische Literatur 64 (2019), H. 1, S. 27-53, hier S. 33-39.

2 Duchhardt, Heinz: Abgebrochene Forschung. Zur Geschichte unvollendeter Wissenschaftsprojekte, Mohr Siebeck, Tübingen 2020.
} 
erachtet wurden. Ihre ,zentrale Frage“ ist diejenige „,nach den Gründen, warum und wie ,gestandene" Wissenschaftler, ihre' Torsi , akzeptierten', wie sie selbst oder die Nachwelt es begründeten, dass es zur angekündigten Fortsetzung nicht mehr kam [beziehungsweise] welche objektiven Gründe es verhinderten“ (S. 5). Die Umsetzung dieses in der Tat originellen Erkenntnisanliegens erfolgt mittels neun durchgehend quellennaher, detaillierter, nüchtern abwägender Einzelstudien, deren Erstellung anhand einer Vielzahl unterschiedlicher Quellen und dennoch verbliebener Quellenlücken sich nachvollziehbarerweise aufwendig gestaltete. Das erste Kapitel nimmt die 1911 im ersten Band erschienene, 1965 nachgedruckte, bahnbrechende Rekonstruktion des mittelalterlich-frühneuzeitlichen Reichskammergerichts aus der Feder des Rechtshistorikers Rudolf Smend (1833-1918) in den Blick. Von der Kritik auch als vielversprechender Beitrag zur Rehabilitierung des im Zuge der preuBisch-protestantischen Machtstaatlichkeit als schwach geschmähten Alten Reiches gewürdigt, blieb die Erstellung des im Vorwort versprochenen zweiten Bandes aus. Dafür macht Duchhardt innere wie äußere Gründe verantwortlich: von Smend mitvollzogene und mitbetriebene Verlagerungen thematisch-inhaltlicher Prioritäten im rechtshistorischen Feld, entsprechende Anpassungszwänge angesichts der Wechselfälle der deutschen Geschichte vom Kaiserreich zu Weimar und dann zum NS-Regime, institutionell-soziale Karrierebedingungen als Medium oder abhängige Variable dieser Entwicklungen, Zeitknappheit aufgrund teils unausweichlicher, teils selbst gewählter anderweitiger Engagements, beginnend bei der akademischen Lehre und Selbstverwaltung. Publikationsmöglichkeiten hätten für die Fortsetzung offenkundig bestanden. Der Theologensohn habe indessen ,ein schlechtes Gewissen, seiner Disziplin den versprochenen zweiten Band vorenthalten zu haben, [...] wohl nur selten empfunden“ (S. 24). Im zweiten Kapitel forscht Duchhardt dem Ausbleiben des Folgebandes der ebenfalls als Pionierarbeit geltenden Darstellung „Russlands Orientpolitik in den letzten zwei Jahrhunderten“ (Band 1, 1913) des Osteuropahistorikers Hans Uebersberger (1877-1962) nach. Hier war das Erscheinen des zweiten Bandes sogar schon für „einige Monate“ nach dem ersten angekündigt (S. 27). Mehr noch, auch für seine thematisch anders gelagerte Habilitationsschrift stellte der Autor eine „nicht allzu ferne“ Fortsetzung in Aussicht, die nie erschien (S. 35). Wie im Fall des Reichskammergerichtswerkes, zeigte sich die Fachkritik an der Vorlage des zweiten Teils der „Orientpolitik“ sehr interessiert und versäumte es nicht, auf die Bedeutung des Werkes auch für die Politik hinzuweisen. Dass der Fortsetzungsband ausblieb, obwohl einer Berufungskommission 1914 bereits die ersten Druckproben vorgelegen haben sollen (S. 46), schreibt Duchhardt plausibel einerseits dem ausgeprägten Engagement des ,eher aus kleinbürgerlichen Verhältnissen“ (S. $27 \mathrm{f}$.) der deutschslowenischen Grenzregion stammenden und daher zweisprachigen Historikers für die NS-Wissenschafts- und Ostpolitik sowie dessen beeindruckend breit angelegter Vertuschung nach $1945 \mathrm{zu}$. Andererseits deutet er anderorts stärker beachtete ,persönlich-emotionale Motive“ - konkret die Liebesbeziehung zu einer jungen Frau, die als Studentin in sein Leben trat - und daraus erwachsene Belastungen an (S. 27 f., 
Fußnote 1). ${ }^{3}$ Über diese Perspektive hinaus besonders frappant sind die Hinweise Duchhardts auf die ,Weichspülung' Uebersbergers nach 1945 durch seine Schüler (S. 41-45). Einen etwas anders gelagerten Fall führt Kapitel drei vor. Der aus einem nichtakademischen, später finanziell angeschlagenen Elternhaus stammende, über das gymnasiale Lehramt, die Habilitation und ein Extraordinariat in eine wichtige Projektleitungsstelle der Bayerischen Akademie aufgestiegene Humanismus- und Reformationsexperte Paul Joachimsen (1867-1930) hatte 1910 im ersten Teil seiner erweiterten Habilitationsschrift zur Geschichtsauffassung und Geschichtsschreibung unter dem Einfluss des (Früh- und Hoch-) Humanismus die Publikation eines zweiten Teils zugesagt. Dass es - möglicherweise nur sehr knapp (S. 62) - nicht dazu kam, dürfte hier einerseits äußerlich den Belastungen geschuldet gewesen sein, die der ungewöhnliche Karriereweg mit sich brachte. Auf der anderen Seite erscheint Duchhardts Vermutung nicht unplausibel, dass auch ein inhaltlicher Grund, konkret Joachimsens negative Einschätzung des noch zu behandelnden Späthumanismus als Dekadenz, eine Rolle spielte. Nochmals anders gelagert war der im vierten Kapitel geschilderte Fall des Nichterscheinens des Fortsetzungsbandes zur Studie „,Der deutsche Staat des Mittelalters" (Band 1 publiziert 1914) von Georg von Below (1858-1927). Der ostpreußische Gutsbesitzer- und Offiziersabkömmling vereinigte, wie Duchhardt konstatiert, monarchisch-konservative bis reaktionäre politische Gesinnung mit methodischem Scharfsinn. Damit vermeidet Duchhardt die heute übliche, einseitige politisch-ideologische Verdammung des durch seine Polemik gegen Karl Lamprecht bekannt gewordenen Historikers. Das wesentlich zeitgeschichtlich vermittelte (S. 75 f.), eigentliche Anliegen des unausweichlich in die Kriegsumstände geratenen Staatsbuches bestand nach eigenem Zeugnis Belows darin, dem Deutschen Kaiserreich eine seit dem Mittelalter hervorgebrachte, kontinuierliche Staatlichkeit zuzuweisen: „Es kann (und darf!) nicht sein, dass Deutschland weniger Staatlichkeit besessen habe als Frankreich oder England“. Während der publizierte erste Band diese ,These [...] in großen Zügen“ entwickelte, sollte der zweite, als Materialsammlung offenkundig bereits vorbereitete, ,die Einzelausführung bringen“ (S. 65f., 87). Mit der auch familiär hautnahen Erfahrung von Niederlage im Weltkrieg und Etablierung einer Republik entfielen jedoch wichtige zeitgeschichtlich-politische Voraussetzungen. Zudem war die psychische und physische Kraft Belows offenkundig zu schwer angeschlagen, um noch mehr als nur einzelne Gedanken und kurze, thematisch verwandte Beiträge vorbringen zu können. In seiner anschließenden ,Zwischenbilanz“ (S. 89f.) schreibt auch Duchhardt dem Umbruch von 1918/19 zu, Relevanzen, Erkenntnisinteressen und Methoden grundsätzlich verschoben zu haben. Hier könnte der aufmerksame Leser einen Verweis einerseits auf Reinhart Kosellecks Arbeiten zur wechselseitigen Abhängigkeit von Zeiterfahrung, Geschichtsauffassung und Methode geschichtlicher Wissenschaft, andererseits zur dramatischen Krise des protestantisch-borussisch [borussianisch ist seit Wolfgang Hardtwigs Aufsatz zum Borussianismus eigentlich der historiografiegeschichtliche Fachbegriff] imprägnier-

\footnotetext{
3 Vgl. dazu ausführlicher Wakounig, Marija: Hans Uebersberger (1877-1962). Eine Gratwanderung. (S)eine Karriere im Fokus privater und öffentlich-beruflicher Spannungen, in: Hruza: Österreichische Historiker, Bd. 3 (wie Anm. 1), S. 157-184.
} 
ten Geschichtsbildes infolge des Untergangs des Kaiserreichs, das als entsprechend ersehnter Modellstaat diente, vermissen. ${ }^{4}$

$\mathrm{Zu}$ einem dezidierten Fazit gelangt Heinz Duchardt bei seinem nächsten Beispiel (Kapitel 5), der Untersuchung des Ausbleibens eines Folgebandes zur „Geschichte der antiken Philosophie“ (Band 1, 1921) des, wie Joachimsen jüdischstämmigen, in Basel wirkenden Philosophen Karl Joel (1864-1934). Dessen „höchst geistvolles opus maximum blieb ein Torso, weil dieser charismatische und ungemein produktive Philosophiehistoriker seine Arbeitskraft schlicht überschätzt hatte und in einer schon von Krankheiten gezeichneten Schaffensperiode nolens volens einem anderen zweibändigen Projekt den Vorzug geben musste“ (S. 112).

Das sechste Kapitel des Buches fällt wiederum in die Wissenschaftsgeschichte der Geschichtswissenschaft im engeren Sinne. Es geht dabei um die offenbar lange geplanten und nachweislich in Angriff genommenen, aber letztlich nicht zustande gekommenen Folgebände von Gerhard Ritters „Geschichte der Universität Heidelberg im Mittelalter“ (Band 1, 1936). Eine fundierte und repräsentative Heidelberger Universitätsgeschichte war von Seiten eben der Universität und der Heidelberger Akademie seit 1914 angestrebt worden, durchaus auch von dem Gedanken motiviert, der protestantischen Wissenschaft Ehre zu verschaffen. 1916 erreichte das Vorhaben über dessen Doktorvater den Pastorensohn und promovierten Gymnasiallehrer Ritter (1888-1967), der 1919 hauptberuflich daran arbeitete, sich aber nach seiner Habilitation 1921 nachvollziehbarer Weise mit anderen Themen befasste und seine Ordinarienkarriere verfolgte. Erst unter ständigem Drängen der Akademie kam dann 1936 der erste Band in Druck, mit vergleichsweise magerem und in zumindest einem Fall sehr kritischem Rezensionsecho, was aber gewiss auch dem damals wie heute vergleichsweise randständigen Thema geschuldet war. Noch im gleichen Jahr bewilligte die Akademie nicht unerhebliche Mittel zur Fertigung des nächsten Bandes und die entsprechenden Arbeiten kamen unter beeindruckendem Hilfskrafteinsatz in Gang, bis der abermalige Weltkrieg mit seinen Dienstverpflichtungen, Archivsperrungen und Papierrationierungen das Ganze beendete. Nach 1945 bekannte sich der Doyen der gemäßigt national-konservativen protestantischen Geschichtswissenschaft zwar „bis ins hohe Alter zu seine[r] sittlichen Verpflichtung“, das Werk fortzuführen oder sogar abzuschließen (S. 138f.). Ernsthaft fordern mochte es jedoch auch die Akademie nicht mehr, und von Ritters Schülern nahm gewiss auch deshalb keiner den Stab auf, weil dadurch kaum akademische oder öffentliche Reputation zu gewinnen war. Dagegen scheint, wie Kapitel 7 darlegt, der deutlich weniger bekannte, katholische Historiker Heribert Raab (1923-1990), tätig als Mitarbeiter der „Germania Sacra" unter anderem in Rom, habilitiert in Mainz und schließlich Ordinarius in Fribourg, auf den angekündigten zweiten Band seiner als Habilitationsschrift begonnenen Biografie des trierischen Kurfürsten Clemens Wenzeslaus von Sachsen (Band 1, 1962) auch ,,aus grundsätzlichen Bedenken“ verzichtet zu haben (S. 155). Die maßgebliche Fachkritik kreidete ihm teilweise vehement an, die Motive und Wege seines Helden allzu katholisch-apologetisch gedeutet zu haben, statt, wie in

\footnotetext{
4 Vgl. umfassend Koselleck, Reinhart: Zeitschichten. Studien zur Historik, Suhrkamp, Frankfurt a.M. 2000, und aus der Debatte zuletzt u. a. Dunkhase, Jan Eike: Absurde Geschichte. Reinhart Kosellecks historischer Existentialismus, Deutsche Schillergesellschaft, Marbach am Neckar 2015.
} 
der damaligen Auftaktphase sozialgeschichtlich-moderner Geschichtswissenschaft en vogue, primär oder ausschließlich auf diesseitig-materielle Interessen abzustellen. Raabs „,nie geschriebener und nie gedruckter zweiter Band“ sei mithin ,einer der wenigen Fälle [...], dass ein problematischer, wenn nicht verfehlter Ansatz nicht nur von der wissenschaftlichen Kritik thematisiert und ,aufgespießt ‘ wurde, sondern zum Wenigsten auch mitverantwortlich dafür war, dass ein Autor einer schwierigen Beweisführung, allen Einwänden zum Trotz doch von der ,richtigen“ These ausgegangen zu sein, aus dem Weg ging und sich einem anderen Forschungsfeld zuwandte“ (S. 158). Kapitel 8 widmet sich dem Ausbleiben eines zweiten Bandes zu „Alles oder Nichts“ (Band 1, 1966), einer ursprünglich vom Bundesverteidigungsministerium angeregten, vom Autor mit diesem ominösen Titel versehenen Geschichte des NS-Staats. Der Verfasser war der ,eher ärmlichen bäuerlichen Verhältnissen entstammende“ (S. 160), in Kiel promovierte und habilitierte, kurz an der NS-Reichsuniversität Straßburg, dann am Mainzer Institut für Universalgeschichte tätige Martin Göhring (1903-1968). Nachdem ein erstes Teilmanuskript bei einem Verlag abgelehnt worden war, musste sich der hier ungeschminkt als ,schwieriger, nicht nur geldhungriger und geltungssüchtiger und in gewisser Weise auch eitler [Verlags-]Klient" charakterisierte Autor geradezu in den Tübinger Mohr Siebeck Verlag einschleichen (S. 163), den er dann ständig mit Reklamieren, Monieren, Lamentieren und Geldforderungen eindeckte (S. 166). Das fachwissenschaftliche und publizistische Echo auch des Auslands auf den schließlich vorgelegten ersten Band erwies sich zudem nicht als übermäßig begeistert, obwohl - derartige Feststellungen finden sich äußerst selten! - „Göhring die Rezensenten (meistens) selbst ,rekrutierte “" (S. 168). ${ }^{5}$ Als ,immerhin gut lesbare Zusammenfassung des Forschungsstandes“ hatte das Werk angesichts der zeitgenössisch neu aufbrechenden NS-Forschung mithin „nur eine begrenzte Halbwertzeit“ (S. 173). Göhring plante und begann offenkundig gleichwohl einen Folgeband, bis ihm - so die aus den Nachrufen bekannte Formel - der unerwartete Tod die Feder aus der Hand nahm (S. 175). ${ }^{6}$ Weder einer seiner Nachfolger und Anhänger noch der Verlag waren zur Überarbeitung und Fertigstellung des Werkes bereit. „Die Fachwissenschaft trug deswegen wohl kaum Trauer“ (S. 178). Duchhardts letztes (9.) Kapitel bezieht sich auf einen für die Frühneuzeitforschung unzweifelhaft noch immer ganz wesentlichen Fall des kurzzeitigen Saarbrücker, dann Bonner katholischen Ordinarius Konrad Repgen (1923-2017): die nicht über zwei erste Teilbände hinausgekommenen Geschichte des Verhältnisses der Römischen Kurie zum Westfälischen Frieden (Band 1 in zwei Bänden 1962/65). Die Anfänge der Studie sind mit Repgens Habilitation in Bonn und dem von seinem akademischen Lehrer Max Braubach, der ,insgesamt weit über 100 Schüler“ an sich binden konnte (S. 183), betriebenen Großvorhaben einer Edition aller wesentlichen Akten des Westfälischen Friedens verknüpft. Dass sie kaum vorwärts kam, ja nicht einmal bis zum Frieden selbst vorstieß, schreibt unser Autor, der als Frühneuzeit-

\footnotetext{
5 Vgl. auch zum corpsstudentisch gestifteten Draht Göhrings zum FAZ-Feuilleton S. 170.

${ }^{6}$ Vgl. exemplarisch Angermann, Erich: Ein abgebrochenes Lebenswerk. Zum Tode Friedrich Hermann Schuberts, in: Historische Zeitschrift 218 (1974), H. 2, S. 354-363. Auf den hier vorliegenden Sonderfall eines Selbstmordes offenkundig auch aus Karrieregründen wäre wissenschaftsgeschichtlich spezifisch einzugehen.
} 
historiker Repgen geradezu unvermeidlich persönlich kannte (S. 194), neben den üblichen und weniger üblichen anderweitigen Verpflichtungen und Umständen vornehmlich dem akribisch-peniblen und daher vergleichsweise langsamen Arbeitsstil schon des akademischen Lehrers und dann dessen „Meisterschülers“ zu (S. 182). Weniger deutlich wird, dass Repgen sich allerdings auch dazu berufen fühlte, der nicht nur aus seiner Sicht immer noch protestantismuslastigen ,Zunft ${ }^{\star}$ auch außerhalb des frühneuzeitlichen Teilgebiets thematisch-inhaltlich, methodisch und im Hinblick auf die Positionsbesetzungen engagiert gegen zu halten, und dadurch manche Energien band. Dass ,einer der großen deutschen Historiker des letzten Drittels des 20. Jahrhunderts“ (S. 179) einen Torso hinterließ, unterstreicht dennoch unzweifelhaft in erster Linie, dass auch objektiv Quellenzugangsproblematik, Quellenfülle und Quellenauswertungsaufwand Projekte scheitern lassen können.

Der knappe „Schluss“ des Bandes (S. 197-205) unternimmt erwartungsgemäß einen ersten Schritt zur vergleichenden Gewichtung der erarbeiteten Befunde auf nächsthöherer Abstraktionsebene. Der angestrebte, nicht repräsentative, aber reflektiert explorative Einblick in ,das breite Spektrum“ (S. 197) der historisch arbeitenden, hinsichtlich ihrer Herkunft, der Konfession (mit ,absoluter Dominanz protestantischer Autoren“ (S. 198)), des Professionstypus (stiller Gelehrter versus ,öffentlicher" Wissenschaftler) und ihrer Lebenswege erfassten Männer (!) hat unzweifelhaft als gelungen zu gelten. Hinsichtlich der Ursachen, die für die Nichtrealisierung von Folgebänden sorgten, fächert die Pionierstudie ein breites Panorama innerer und äußerer Gründe auf, ohne den augenfälligen wesentlichen äußeren Grund im beobachteten Zeitraum, die Weltkriege und ihre massiven Einschnitte, übermäßig zu strapazieren. Soziale Herkunft, Konfession, vielleicht auch Alter spielten eine eher geringe Rolle. Duchhardt meint immerhin eine relative Häufung aufgegebener Projekte in der Rechtsgeschichte feststellen zu können. Dies sei dadurch bedingt, dass die praktische Juristenausbildung den Verfassern überdurchschnittliche Energien abverlangte (S. 202f.). Am Ende folgen kritische Verweise auf die Quellenlage, insbesondere die schlechte Überlieferung von Verlagsakten, schließlich eine Reihe weiterführender, unverkennbar auch persönlich inspirierter Fragen, die sich einerseits wieder auf die reputativen Konsequenzen gebrochener Fortsetzungsversprechen im Rahmen der ,Zunft' und der Öffentlichkeit, andererseits aber - und das noch deutlicher - auf die mentale Verarbeitung dieses Mangels bei den Autoren selbst beziehen: ,das Gefühl, eine Bringschuld nicht beglichen zu haben“; die Notwendigkeit, „solche Verlustängste“ zu bewältigen, wobei die Untersuchung solcher Bewältigungsformen nicht mehr in die Kompetenz des Historikers fielen (S. 205). ${ }^{7}$

Das Potenzial dieser Kollektion individualbiografischer Studien für eine systematisch konzipierte Wissenschaftsgeschichte der Geschichtswissenschaft ist beträchtlich, wie schon ein kurzer Blick auf dieses von anderen Autoren durchaus betretene Feld unterstreicht.

Die vorliegenden Ausführungen beziehen sich wie gesagt zunächst auf die soziale Herkunft, die damit verbundene konfessionelle und akademische Sozialisation sowie den Karriereverlauf der erfassten Autoren. Deren Ausprägungen wären denjenigen

\footnotetext{
${ }^{7}$ Der Kulturhistoriker wird sich der genannten Bewältigungsformen vermutlich doch etwas genauer annehmen. Für das angefügte, zuverlässige Personenregister gebührt Autor und Verlag besonderer Dank.
} 
Befunden zuzuordnen, die für die gesamte Historikerzunft und den angelagerten historischen Sonderrichtungen (zum Beispiel eben Rechtsgeschichte) erarbeitet worden sind. Der Herkunft im engeren sozialen Sinne, gar aufgefasst als ,Klassenherkunft", wird man heute - wie von Duchhardt bereits angedeutet - indessen keine übermäßige Bedeutung für die Wissenschaftsauffassung und -praxis mehr zuschreiben, während dem konfessionellen Faktor eher wieder größere Wichtigkeit zugesprochen wird, vor allem hinsichtlich der Komponenten Berufseifer, Berufsethos (siehe unten), Deutungshorizonte und Themenwahl beziehungsweise thematische Prioritätensetzung. ${ }^{8}$ Hinzu kommt der vorliegend sachbedingt ausgeblendete, aber notierte Genderaspekt, für den nunmehr die beeindruckende Studie von Falko Schnicke vorliegt. ${ }^{9}$ Mit dem Aspekt der akademischen Sozialisation eng verknüpft wird aktuell nicht nur die Einübung in das oder - böse ausgedrückt - die Abrichtung zum Historikergeschäft, sondern insbesondere der Erwerb oder die Stabilisierung von sozialen Beziehungen, vor allem in Form der akademischen Schülerschaft. Zwar sind Kontinuität und Wandel dieser partiell tabuisierten wissenschaftlich-disziplinären Sozialstruktur zumindest seit 1970 noch unklar. Um sie näher auszuleuchten, ,bedürfte es eines umfassenden Projekts zur Genealogie der deutschen Historikerzunft [...], das die Pionierstudie von Wolfgang Weber über die ,Priester der Clio' fortführen würde“. ${ }^{10}$ Dass die Struktur ,Schule “ - wobei zwischen direkten und indirekten, ,Kurz- ' und ,Langschülern“ et cetera zu unterscheiden wäre - und die mehr oder weniger fluiden Zweckzusammenschlüsse von Schulen (action groups) bereits einem freien Markt geschichtswissenschaftlicher Einzelkämpfer gewichen seien, deren Karrieren allein von ihrer persönlichen Performanz, argumentativen Brillanz und soziokulturellen Passform abhängten, ist jedoch ziemlich unwahrscheinlich. Schulen von mehr oder weniger nahestehende Kollegen und Weggefährten einerseits sowie der Lagerbildung in der weiteren Öffentlichkeit andererseits zu unterscheiden erscheint besonders auch im Hinblick auf Duchhardts Leitfrage nach der moralischen Verpflichtung beziehungsweise dem Sich-Verpflichtet-Fühlen wichtig. Wem genau gelten diese Verpflichtungen, ist das Vorbringen von Entschuldigungsgründen adressatenspezifisch aufzufächern, ist ein ,Versagensgeständnis ‘ vor dem akademischen Ziehvater oder den eigenen Schülern besonders peinigend? Oder gehören schnelle In-Aussicht-Stellungen oder Zusagen heute lediglich zum unausweichlichen Selbstvermarktungsgeschäft? Auch der damit angesprochene Sonderbereich der wissenschaftlichen beziehungsweise akademischen (fach- und fakultätsspezifischen, universitären) und allgemein

\footnotetext{
${ }^{8}$ Ein gewisser Umbruch, d.h. eine (vorübergehende?) Abschwächung der protestantischen Imprägnierung, scheint um 1965 stattgefunden zu haben, vgl. Weber, Wolfgang E.J.: „Es ist eine merkwürdige Tatsache, daß die protestantische Leben-Jesu-Forschung viel weniger apologetisch war und ist als die Lutherforschung“. Lutherbild und Geschichtswissenschaft im 19. und 20. Jahrhundert, in: Richard Faber, Uwe Puschner (Hrsg.): Luther zeitgenössisch, historisch, kontrovers (Zivilisationen \& Geschichte, Bd. 50), Peter Lang, Frankfurt a.M. u. a., 2017, S. 317-342.

9 Schnicke, Falko: Die männliche Disziplin. Zur Vergeschlechtlichung der deutschen Geschichtswissenschaft 1780-1900, Wallstein, Göttingen 2015.

10 Nonn, Christoph: Der Meister, die Methode und die Politik. Theodor Schieder und seine Historikerschule, in: INDES 3 (2014), H. 3, S. 87-94, hier S. 92; Weber, Wolfgang E.J.: Priester der Klio. Historisch-sozialwissenschaftliche Studien zur Herkunft und Karriere deutscher Historiker und zur Geschichte der deutschen Geschichtswissenschaft 1800-1970, Peter Lang, Frankfurt a. M. u. a. 1984. Vor einem Anschlusswerk müsste auch dieses Buch gründlich an den aktuellen Forschungsstand angepasst werden.
} 
(bildungs-)bürgerlichen Ethik (etwas weniger prätentiös: Anstand, Comment) ${ }^{11}$ ist bereits in den Fokus geschichtswissenschaftshistorischer und allgemein geisteswissenschaftshistorischer Forschung gerückt. An der Universität Leiden hat sich um eine entsprechend denominierte Professur ein einschlägiger Schwerpunkt und eine entsprechende Zeitschrift (History of Humanities) etabliert. Der Inhaber dieser Professur, Paul J. Herman, ist mit Studien etwa zum Selbstverständnis und den daraus abgeleiteten professionellen Normen der ,scholarly persona“ und der ,,scholarly integrity“ - mit Schwerpunkt auf Historikern des 19. und frühen 20. Jahrhunderts hervorgetreten. ${ }^{12}$ Andere Ansätze operieren mit dem analytischen Begriff „tribal culture“, woran sich die Herkunft dieser analytischen Perspektiven aus der Kulturwissenschaft ablesen lässt. ${ }^{13}$ In die mithin deutlich zu differenzierende Beurteilung gebrochener Zusagen wird man ferner - freilich angemessen kritisch - die Kategorie ,wissenschaftliches Fehlverhalten ' einbeziehen müssen. ${ }^{14}$ Im Übrigen scheint auch die Berücksichtigung anderer Phänomene unvollendeter, nichtrealisierter oder revidierter Projekte und Werke in der Geschichtswissenschaft erforderlich, um die von Heinz Duchhardt untersuchten Fälle angemessen beurteilen zu können. Das aufgehobene Max Planck Institut für Geschichte hat ,in der Ära Heimpel“ laut Peter Schöttler keines seiner großen Langzeitvorhaben abgeschlossen. ${ }^{15}$ Die erste Auflage der Edition der Briefe Leopold von Rankes, immerhin unter der Ägide der Bayerischen Akademie der Wissenschaften, wurde wegen erheblicher Mängel eingestampft und soll durch eine Neuauflage ersetzt werden. ${ }^{16}$ Ein möglicherweise spannendes Thema wäre etwa auch, zu untersuchen, welchen Publikationsverpflichtungen Stipendiaten oder sonstige Geldempfänger bestimmter Stiftungen oder Mäzene aus welchen

${ }^{11}$ In diesen Bezug ist zeitgenössisch der o. a. Fall Uebersberger(s) gestellt worden, sich mit einer Studentin eingelassen zu haben.

12 Exemplarisch Paul, Herman J.: Friedrich Christoph Dahlmann and Georg Waitz: Two Models of Scholarly Integrity, in: C. Alston u.a. (Hrsg.): Portraits of Integrity. 26 Case Studies from History, Literature and Philosophy, Bloomsbury, London 2020, S. 207-215; Ders.: A Missing Link in the History of Historiography. Scholarly Personae in the World of Alfred Dove, in: History of European Ideas 45 (2019), H. 7, S. 1011-1028; Ders.: The Virtues of a good Historian in Early Imperial Germany: Georg Waitz's contested Example, URL: www.cambridge.org/core/journals/modernintellectual-history/article/abs/virtues-of-a-good-historian-in-early-imperial-germany-georg-waitzscontested-example/367D62B4D48A58FD6A59BD2FB91665E2 [Zugriff: 08.08.2020]. Die genannte Zeitschrift ist offenbar in den wissenschaftlichen Bibliotheken der Bundesrepublik Deutschland bisher noch so gut wie gar nicht vorhanden.

${ }^{13}$ Noch immer wichtig Becher, Tony: Academic Tribes and Territories. Intellectual Enquiry and the Culture of Disciplines, Open UP, Stony Stratford 1989; überarbeitete, von Paul R. Trowler mitverfasste zweite Auflage 2011.

${ }^{14}$ Siegel, Stefan T., Daumiller, Martin H. (Hrsg.): Wissenschaft und Wahrheit. Ursachen, Folgen und Prävention wissenschaftlichen Fehlverhaltens, Budrich, Opladen 2020.

15 Schöttler, Peter: Das Max-Planck-Institut für Geschichte im historischen Kontext 1972-2006. Zwischen Sozialgeschichte, Historischer Anthropologie und Historischer Kulturwissenschaft, Preprint 15, Berlin 2020, S. 36.

${ }^{16}$ Muhlack, Ulrich/Ramonat, Oliver (Hrsg.): Gesamtausgabe des Briefwechsels von Leopold von Ranke, Bd. 1: 1813-1825, Oldenbourg, München 2007. Vgl. die trotz einiger zustimmender Fachrezensionen nicht sonderlich beachtete, ebenso fundierte wie nüchtern-kritische Stellungnahme und Materialsammlung des promovierten Historikers, jedoch außerfachlich tätigen Experten Henz, Günter Johannes: Leopold von Ranke in Geschichtsdenken und Forschung, Bd. 1: Persönlichkeit, Werkentstehung, Wirkungsgeschichte. Bd. 2: Grundlagen und Wege der Forschung, Duncker \& Humblot, Berlin 2014. 
Gründen nicht nachgekommen sind oder nicht nachkommen konnten, und welche Konsequenzen die etwaigen Verfehlungen gegebenenfalls hatten.

Ein Ort, indem das disziplinäre Ethos regelmäßig besonders prägnant zum Ausdruck kommt, sind die von Duchhardt angesprochenen Rezensionen, die eben keineswegs nur der Wissenschaft in ihrer kognitiven Verfassung dienen, sondern auch ihrer sozialen Verfassung zugehören. Erwähnt sei nur der triviale Tatbestand, dass Rezensionen nicht nur über Buch-, sondern auch über Wissenschaftlerkarrieren entscheiden können. Eine umfassende historische Untersuchung der Textsorte Rezension in der Geschichtswissenschaft sowohl in deren kognitiver als auch sozialer Dimension gibt es bis heute ebenso wenig wie eine profunde Ausbildung künftiger Historiker in dieser fachwissenschaftlich zentralen Textsorte, von löblichen Ausnahmen abgesehen. ${ }^{17}$ In diese künftige Forschung wären sämtliche von unserem Mainzer Autor explizit und implizit aufgeworfenen Fragen einzubeziehen: Wer bespricht wie ein umfangreiches oder ambitiöses Werk eines hoch angesehenen Fachvertreters in welchem führenden oder eben im Gegenteil eher als weniger bedeutend erachteten Fachjournal? Wer darf eine solche Besprechung schreiben? Wie kommt überhaupt der Abdruck einer Rezension, wie die Auswahl der Rezensenten (siehe den Fall Martin Göhring) zustande? Welche Art, welcher Grad von Kritik erscheinen zulässig, nach welchen Kriterien hat diese Kritik zu erfolgen? Wie sieht es mit den hier und da mündlich beiläufig verdammten Besprechungskartellen - positive Rezensionen auf Gegenseitigkeit - im Fach Geschichte aus?

Nachdem die Rezension über ihre unmittelbare Aufgabe hinaus eine zentrale Funktion in der fachlichen und außerfachlichen Wissenschaftskommunikation insgesamt einnimmt, ist schließlich auch diese Kommunikation, die gleichzeitig die soziale Vernetzung konstituiert, systematisch in den Blick zu nehmen. Das gilt einerseits für die fachwissenschaftlichen Medien: die Bedeutung und der Wandel etwa der Historischen Zeitschrift für das Gesamtfach, der Geschichte und Gesellschaft für die Bielefelder Schule, das äußere (finanziell-ökonomische) Scheitern einer Spezialzeitschrift wie derjenigen, an der Heinz Duchhardt selbst beteiligt war. ${ }^{18}$ Andererseits kommen die angesichts von Globalisierung und Europäisierung eher beachteten Studien zum internationalen und nationalen Tagungswesen des Faches Geschichte hinzu. ${ }^{19}$ Jüngere Individualbiografien zu Historikern weisen eigene Abschnitte zu den kommunikativen und sozialen Vernetzungen ihrer Helden aus. Paul Nolte hat unlängst eine eindrucksvolle Pionierstudie zur Entstehung eines einzelnen, ,zünftig ‘

\footnotetext{
17 Vgl. zur jüngsten Inaugenscheinnahme der historischen Rezension unter dem Gesichtspunkt der Evaluationsforschung die Beiträge zu Schweizerische Zeitschrift für Geschichte 62 (2012), H. 2: Qualität der Forschung, insbesondere Lengwiler, Martin: Geschichtswissenschaften in the making. Skizze einer Praxisanalyse historischer Forschung, in: Schweizerische Zeitschrift für Geschichte 62 (2012), H. 2, S. 268-286; einen Einblick in die Rezensionskultur der Historischen Zeitschrift habe ich versucht in Weber: Tatsache (wie Anm. 8).

18 Duchhardt: Forschung (wie Anm. 2), S. 204f. Die einschlägigen historischen Rückblicke in den Jubiläumsbänden der Historischen Zeitschrift könnten teilweise durchaus kritischer sein. Zur Geschichte der historischen Fachjournale noch immer grundlegend Middell, Matthias (Hrsg.): Historische Zeitschriften im internationalen Vergleich, Akademische Verlagsanstalt, Leipzig 1999.
}

19 Zuletzt mit allen weiteren Verweisen Berg, Matthias u. a.: Die versammelte Zunft.

Historikerverband und Historikertage in Deutschland 1893-2000. 2 Bde., Wallstein, Göttingen 2018. 
noch immer höchst angesehenen historischen Werkes vorgelegt, dem sich nicht nur komplexe kognitive Denkprozesse, beflügelte und resignative Arbeitsphasen und nicht zuletzt Abbruchgefahren, sondern auch entsprechende Kommunikationsprozesse zumal zum Verleger entnehmen lassen. ${ }^{20}$ Dagegen fehlen systematische und nüchterne Analysen etwa zum Begutachtungswesen. Die Bedeutung dieses Verfahrens für die kognitive und soziale Reproduktion und Innovation der Disziplin dürfte kaum jemand in Abrede stellen. Gutachterliche Stellungnahmen entscheiden bereits über Publikationschancen, also die Eintrittskarten in das akademische Geschäft schlechthin, auch wenn aktuell und in absehbarer Zukunft offenbar nicht die fachwissenschaftliche Expertise, vielmehr ökonomisch-finanzielle, also markt- beziehungsweise absatzbezogene Erwägungen und Entscheidungen der Verlegerseite in den Vordergrund rücken. Noch wesentlicher sind Projektbegutachtungen, gefolgt von Personalgutachten, dem nach wie vor am stärksten tabuisierten, dabei gerade relevantesten Feld. ${ }^{21}$ Welche Evaluationskriterien wurden und werden wann in welcher Gewichtung und in welchen Formulierungen angesetzt? Wie sieht es neben den problematischen Kriterien ,Leistung ‘ und ,Wirkung/Erfolg (impact) ‘ mit dem in Berufungsprozessen oft umschriebenen, also nicht explizit erörterten Kriterium ,Passfähigkeit‘ aus? Diese kann sich ja auf ganz verschiedene Elemente beziehen, so das Fach, die Fakultät, die Universität, die Region und ähnliches, aber auch inhaltlich-thematische Nähe (welche Schwerpunkte werden vertreten) und soziale Beziehungen (die berühmte ,Chemie' muss stimmen). Auch hier spielen unaufhebbar das von Duchhardt betonte Ethos, die Selbstauffassung, das Selbstwertgefühl, die Integrität der betroffenen, wissenschaftlichen Person' und der Beteiligten eine zentrale Rolle. Dass gegen Lebensende auch bei denjenigen, die Berufungsprozesse glücklich überstanden, leicht Pessimismus, Resignation und Vergeblichkeits-Anwallungen auftreten können, sei zum Abschluss wenigstens anhand eines Zitats belegt: Johannes Haller, der konservativ-nationale Historiker, meinte im Mai 1947, kurz vor seinem Tode im Dezember des gleichen Jahres, einem Freund folgende Bilanz anvertrauen zu müssen: „Die Rückschau auf meinen langen Lebensweg stimmt mich vollends herab. Lauter Anfänge, denen der Abschluss fehlt, nichts Ganzes, u. nirgends ein durchschlagender Erfolg! Die wissenschaftliche Richtung, für die

\footnotetext{
20 Nolte, Paul: Lebens Werk. Thomas Nipperdeys Deutsche Geschichte. Biographie eines Buches, C.H. Beck, München 2018; vgl. die abschließenden Notate zum Steckenbleiben großer Projekte bei Theodor Mommsen, Heinrich von Treitschke und Franz Schnabel, S. 312. Zum Gelingen dieses Buches hat nach eigener Aussage des Verfassers auch beigetragen, dass er Thomas Nipperdey ,persönlich nie begegnet" (S. 313) ist, also nicht in ein ethisches Dilemma aufgrund persönlicher Beziehung geriet.

21 Natürlich gehen Gutachtenauswertungen auch in - soweit rechtlich möglich - sämtliche individualbiographische und institutionengeschichtliche Historikeranalysen ein; was fehlt, sind aber eben eigenständige, systematische Studien hinsichtlich Sprache, Einsatz- bzw. Umgangsformen etc. im historischen Wandel. Wenn ich richtig sehe, bewegen sich bestimmte soziologische Forschungen am stärksten in diese Richtung, die eigene ,Zunft', also sich selbst, zum Forschungsgegenstand zu machen, vgl. Dressel, Gert/Langreiter, Nikola: Wenn ,wir selbst“ zu unserem Forschungsfeld werden, URL: www.qualitative-research.net/index. php/fqs/article/view/700/1517 [Zugriff: 21.07.2020]; Hamann, Julian: Wie entstehen wissenschaftliche Subjekte?, in: Ders. u. a. (Hrsg.): Macht in Wissenschaft und Gesellschaft. Diskurs- und feldanalytische Perspektiven, Springer VS, Wiesbaden 2017, S. 83-111; Ders.: Berufungsakten als Datensorte für die Geschichte der Soziologie, in: Moebius, Stefan/Ploder, Andrea (Hrsg.): Handbuch Geschichte der deutschsprachigen Soziologie, Bd. 2: Forschungsdesign, Theorien und Methoden, Springer VS, Wiesbaden 2017, S. 243-255.
} 
ich gekämpft habe - mit Kritik u. eigenen Leistungen - ist nicht anerkannt, findet keine Fortsetzung, meine Figur ist $u$. bleibt die eines zu Paradoxien geneigten Outsiders, u. es kommt vor, dass die Studierenden vor mir gewarnt werden. Der ,Kollege“ von dem ich weiss, daß er das tut, soll jetzt auf das Katheder berufen werden, das bis vor 15 Jahren das meine war. Ich denke, das genügt, mir das Vergebliche meiner wissenschaftlichen Lebensarbeit zu bescheinigen".22

In summa: Die Wissenschaftsgeschichte der Geschichtswissenschaft ist im Aufbruch begriffen und soll es sein, auch wenn ihre Fragen und Befunde möglicherweise zum Abbruch etablierter Tabus und Vorstellungen führen. Worauf es dabei ankommt, zeichnet sich unverkennbar bereits ab. Die zu untersuchenden Historiker sind nicht mehr bevorzugt als mehr oder weniger geniale, individuelle Geistesheroen ohne wesentliche eigene Bedürfnisse und Interessen zu betrachten, sondern als nach Vernetzung, Entscheidungschance, Einfluss und Reputation je spezifisch positionierte soziale Akteure in ihren wechselnden, bis vor kurzem zunehmend verdichteten disziplinären (und außerdisziplinären) Konstellationen. Dieser Perspektivwechsel öffnet den Blick für alle diejenigen Bedingungen, die das Denken und Handeln strukturell wie situativ eigentlich bestimmen, und ermöglicht deren je spezifische Analyse: Motive, Lektüren, Rezeptionen, Interaktionen, Wahrnehmungen, Gepflogenheiten, Werte, Theorien, Erwartungen, Ideale, Interessen und so weiter. Die Bandbreite der wissenschaftsgeschichtlich wesentlichen (und legitimen) Themen erweitert sich dadurch erheblich. Gleichzeitig werden Quellenwahl und Quellenauswertung komplexer und schwieriger. Nur auf diese Weise lassen sich meines Erachtens jedoch erkenntnishemmende Grundstrukturen wie zum Beispiel Autoritätsgläubigkeit und -fügsamkeit, Innovationsabneigung oder überzogene Fachräson (Intransparenz und Zensur zwecks fachlicher Reputations- und Existenzsicherung) kritisch fassen und überwinden.

\section{Besprochene Literatur}

- Duchhardt, Heinz: Abgebrochene Forschung. Zur Geschichte unvollendeter Wissenschaftsprojekte, 221 S., Mohr Siebeck, Tübingen 2020.

Funding Open Access funding enabled and organized by Projekt DEAL.

Open Access Dieser Artikel wird unter der Creative Commons Namensnennung 4.0 International Lizenz veröffentlicht, welche die Nutzung, Vervielfältigung, Bearbeitung, Verbreitung und Wiedergabe in jeglichem Medium und Format erlaubt, sofern Sie den/die ursprünglichen Autor(en) und die Quelle ordnungsgemäß nennen, einen Link zur Creative Commons Lizenz beifügen und angeben, ob Änderungen vorgenommen wurden.

Die in diesem Artikel enthaltenen Bilder und sonstiges Drittmaterial unterliegen ebenfalls der genannten Creative Commons Lizenz, sofern sich aus der Abbildungslegende nichts anderes ergibt. Sofern das betreffende Material nicht unter der genannten Creative Commons Lizenz steht und die betreffende Handlung

22 J. Haller an K. Straube vom 25.05.1947, in: Zielinski: Johannes Haller und Karl Straube (wie Anm. 1), S. 454-462, hier S. 457. Ein schwerer Verstoß gegen das Fachethos war es demnach zumindest damals, vor einem bestimmten ,Kollegen“ vorlesungsöffentlich zu warnen. 
nicht nach gesetzlichen Vorschriften erlaubt ist, ist für die oben aufgeführten Weiterverwendungen des Materials die Einwilligung des jeweiligen Rechteinhabers einzuholen.

Weitere Details zur Lizenz entnehmen Sie bitte der Lizenzinformation auf http://creativecommons.org/ licenses/by/4.0/deed.de.

Wolfgang E.J. Weber Geb. 1950, Studium in Freiburg i.B., Promotion und Habilitation in Augsburg. Nach Gastprofessur an der Emory University in Atlanta/Georgia und Lehrstuhlvertretungen u. a. in Freiburg i.B. bis 2015 Prof., Wiss. Sekretär und Direktor des Instituts für Europäische Kulturgeschichte der Universität Augsburg. 